\title{
Multi-colour Electron Microscopy: Protein Accumulation and Cellular Activity Surrounding Hydroxyapatite Implants Revealed by Energy Dispersive X-ray Spectrometry
}

\author{
Louise Hughes $^{1}$, Rachel Vowden ${ }^{2}$ and Zhidao $\mathrm{Xia}^{3}$
}

${ }^{1}$ Oxford Instruments NanoAnalysis, High Wycombe, England, United Kingdom, ${ }^{2}$ Oxford Biomedica, Oxford, England, United Kingdom, ${ }^{3}$ Swansea University, Swansea, Wales, United Kingdom

The interaction of biomaterials and implants with cells and tissues remains a critical issue for patients and researchers involved in the biomedical field. Key research trends include the development of multimodal imaging and contrasting mechanisms to facilitate the visualisation of biological samples and biomaterials/implant materials within the same sample [1]. Energy dispersive $\mathrm{x}$-ray spectrometry (EDS) provides elemental contrast and analytical capabilities within an electron microscope, supplying an opportunity for researchers to analyse ultrastructure and composition within the same session. We used EDS on two research topics investigating tissue interactions with implants and biomaterials.

1. The analysis of wear particles from patient tissue and in vitro studies on cultured macrophages.

2. The degradation of hydroxyapatite implants in vivo.

Patient samples were prepared for routine TEM pathology examination. RAW macrophages were seeded onto coverslips in media containing combinations of materials in the form of nanoparticles for 24 hours before being fixed (Karnovsky's fixation) dehydrated, critical point dried and coated with carbon. Hydroxyapatite implant tissue samples were fixed, embedded in resin and ground to a thickness of approximately $1 \mathrm{~mm}$ before being stained using uranyl acetate and lead citrate.

EDS data was collected with an Ultim Extreme detector on an SEM using accelerating voltages of between 5 and $10 \mathrm{kV}$ and a beam current of 1 na or less. Data was collected either as single area scans or using large area mapping in AZtec.

EDS provided a detailed analysis of wear particles, identifying their composition, and localised nanoparticles within cultured macrophages. The compositional analysis demonstrated that wear particles generated from different regions of the implant were located within the same cells.

EDS was instrumental in the identification of cells within the hydroxyapatite implant, showing the location of osteoclasts and osteoblasts within voids and within the implants itself during degradation, which had not been possible to determine using electron data alone (fig. 1). EDS was also used to determine regions of new bone growth and a variation in nitrogen concentration, often used as a marker for peptides [2].

In both studies EDS provided extra image information that facilitated interpretation of structure in addition to biologically relevant compositional information. 


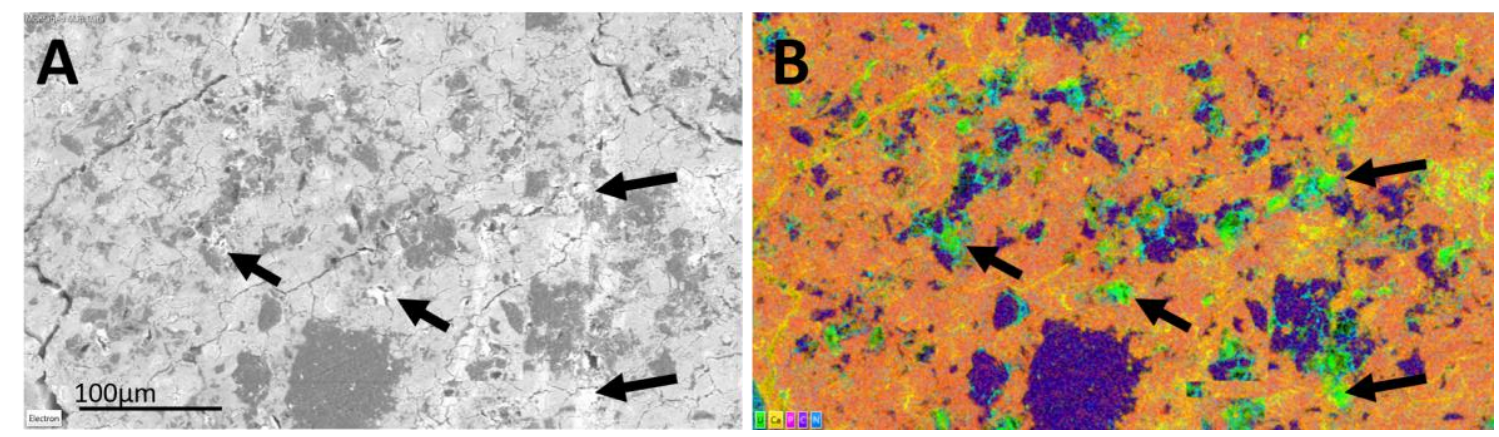

Figure 1. Backscattered electron image (left) and layered EDS maps (right) showing signals from uranium (green), calcium (yellow), phosphorous (pink), carbon (purple) and nitrogen (blue). The uranyl acetate stained cells appear distinct in the EDS maps compared to electron data alone (arrows).

\section{References}

1. Zhang YS, Yao J. (2018) Imaging Biomaterial-Tissue Interactions. Trends Biotechnol. 36(4):403-414 2. Pirozzi, N.M., Hoogenboom, J.P. \& Giepmans, B.N.G. (2018) ColorEM: analytical electron microscopy for element-guided identification and imaging of the building blocks of life. Histochem Cell Biol 150, 509-520 